\title{
Low Dietary Magnesium Intake and Hypertension
}

\author{
Lindsy Kass, Keith R. Sullivan \\ School of Life and Medical Sciences, University of Hertfordshire, Hatfield, UK \\ Email: 1.s.kass@herts.ac.uk
}

How to cite this paper: Kass, L. and Sullivan, K.R. (2016) Low Dietary Magnesium Intake and Hypertension. World Journal of Cardiovascular Diseases, 6, 447-457. http://dx.doi.org/10.4236/wjcd.2016.612048

Received: July 21, 2016

Accepted: December 4, 2016

Published: December 7, 2016

Copyright $\odot 2016$ by authors and Scientific Research Publishing Inc. This work is licensed under the Creative Commons Attribution International License (CC BY 4.0).

http://creativecommons.org/licenses/by/4.0/

\begin{abstract}
Purpose: Magnesium $(\mathrm{Mg})$ is a key factor in blood pressure regulation. However, only in recent years, magnesium dietary intake has been studied in relation to hypertension, with equivocal conclusions. Further no comparisons have previously been made between the UK general population and primary hypertensives, the UK RNI and the USARDA. Methods: Twenty-five hypertensives (HT) (mean age 63.4 y) and twenty-one normotensives (mean age 46.7 y) were recruited from the same geographical area. Food diaries were completed and analysed to determine average daily $\mathrm{Mg}$ intake. $\mathrm{Mg}$ intake was compared between the observed group (OB), normotensives (NT) and general population (GP) and both the UK RNI and the USA RDA. Results: Study participants had a significantly lower dietary Mg intake than the UK RNI ( $p<0.05)$ and the US RDA ( $p<0.05)$. Intake for HT males was significantly lower ( $\mathrm{p}<0.0001)$ than the external control (general population) and, for HT females, intake was significantly lower than the NT $(p=0.006)$. The findings also suggest that with ageing there is a reduction in daily dietary $\mathrm{Mg}$ intake. Finally, when UK external controls were compared to the USA RDA for both males and females they were found to be around 35\% and 30\% respectively below the recommended values. Conclusions: Daily $\mathrm{Mg}$ intake in hypertensives is lower than the general population, the UK RNI and the USA RDA. Daily magnesium intake reduces with age. These findings suggest that low Mg dietary intake increases the risk of hypertension.
\end{abstract}

\section{Keywords}

Magnesium, Hypertension, UK RNI, USA RDA, Blood Pressure, Dietary Intake

\section{Introduction}

Increasing evidence suggests a key role for magnesium in the regulation of blood pressure [1] [2] and a deficiency of it has been demonstrated to contribute to a number of disease states such as type 2 diabetes, metabolic syndrome, elevated $\mathrm{C}$ reactive protein 
and hypertension [3]. Observational studies have shown an inverse association between dietary magnesium intake and blood pressure (BP) [2] [4] [5]; however, a number of meta analyses examining magnesium supplementation have produced conflicting results [1] [6] [7] [8] [9] [10]. Experimental studies have suggested that a deficiency in magnesium may have detrimental effects on blood pressure and the cardiovascular system, and previous studies have examined the effect of magnesium supplementation on hypertension [11] [12] [13] yet there are limited studies that look at long term dietary intake and association with HT. A study by Chidambaram (2014) [14] looked at the Indian population and divided participants into normotensive and hypertensive groups and found that a reduction in $\mathrm{Mg}$ consumption was associated with increased blood pressure. However, this study looked only at urinary biomarkers and not food intake. Choi and Bae (2015) [15] investigated Mg intake in Koreans. The participants were grouped into quartiles of magnesium intake and BP assessed. Women were found to have a negative relationship between magnesium intake and HBP. However, this study did not include those on HT tablets or those with previously diagnosed HT. This current study aims to look at the association between habitual dietary Mg intake and those with existing HT.

Magnesium is the fourth most abundant cation in the body, involved in more than 300 enzymatic systems [16]. Total body stores vary between $21-28 \mathrm{~g}$ in the average 70 $\mathrm{kg}$ adult with most of the magnesium being stored in the bone mass, the remainder is found in soft tissue with only $0.3 \%$ in extra-cellular stores [16], this makes magnesium measurement difficult and is the reason why dietary magnesium intake is often the best measure of hypomagnesia.

Elevated blood of $\mathrm{Mg}$ can cause vasodilation and reduce vasoconstriction of the vasculature in common with other minerals such as $\mathrm{Ca}^{2+}$, a key contributor to vascular smooth muscle constriction [17] [18]. Magnesium exhibits a pharmacological profile comparable to a synthetic calcium channel antagonist; therefore, it has been suggested to be nature's "physiological calcium blocker" [19].

Few intervention studies have examined the effect of habitual dietary magnesium intake on blood pressure. Furthermore, there have been no assessments of habitual dietary magnesium intake in those clinically diagnosed as hypertensive. Therefore, the aim of this investigation is to determine if there is an association between habitual dietary magnesium intake and hypertension.

A study by Ohira et al., (2009) [20] attempted to determine whether there was a correlation between dietary magnesium intake and serum magnesium concentrations in relation to CVD risk. They found that individuals with both dietary and serum magnesium greater than the median had an approximately $35 \%$ lower risk of ischemic stroke.

The Honolulu heart study undertaken by Joffres, Reed and Yano, (1987) [21] demonstrated an inverse relationship between dietary magnesium supplementation and both systolic (SBP) and diastolic blood pressure (DBP). When dietary Mg without additional supplementation was studied, results were similar although the link with DBP 
was reduced $(r=0.6)$. In contrast, a cross-sectional study using data from the National Health and Nutrition Examination Survey (NHANES) [22] reported no significant correlation between magnesium and blood pressure but a limitation of the study was the fact it did not allow for directional or causal influences. The absence of firm consensus despite a demonstrated mechanistic link between the blood pressure reducing action of Mg suggests that dietary Mg intake is worthy of further investigation.

The current RNI in the UK is $300 \mathrm{mg}$ and $270 \mathrm{mg}$ daily for males and females respectively from $18-75+$ years [23]. For the USA the Recommended Dietary Allowance (RDA) is $400 \mathrm{mg}$ and $310 \mathrm{mg}$ for males and females respectively from ages 19 - 30 years and 420 - $320 \mathrm{mg}$ daily for those aged $>31$ years old [24]. The National Diet and Nutrition Survey [23] states that the average consumption of the RNI in the UK is at $90 \%$ for males and $82 \%$ for females, representing a population consuming less than recommended. This study therefore sets out to determine whether those with primary hypertension habitually eat a diet that is both lower than the RNI and lower than the UK national average for age and sex. Further consideration will be given to how this compares to the USA RDA with a substantially higher recommended intake. As the UK national average intake is already lower than the RNI these will be compared to the higher RDA used in the USA to observe the greater difference between intake in the UK and recommendation in the USA.

According to Long Term Health Conditions [25] 1 in 4 adults is affected by high blood pressure in the UK. Figures show that by reducing the blood pressure of the nation as a whole, $£ 850$ million of NHS and social care costs could be avoided over 10

years. Further, if $15 \%$ more people currently being treated for high blood pressure could control it better a further $£ 120$ million could be saved [25]. To date there have been no studies investigating the dietary intake of those with hypertension with regard to magnesium intake from habitual dietary intake.

\section{Materials and Methods}

Twenty-five participants (female $\mathrm{n}=11$, male $\mathrm{n}=14$, mean age $63.4 \pm 10.2 \mathrm{y}$ ) were recruited from the East and North Herts NHS Trust. Ethical approval was granted by North and East Herts Local Research Ethics Committee. Individuals already diagnosed with primary hypertension (and not hypertension caused by either other medical conditions or drug intervention) were identified by a Consultant Cardiologist and were recruited as subjects. Written informed consent was obtained during their appointments with the cardiologist. Thirty-three subjects were recruited with 8 excluded for noncompletion.

A further 21 normotensive participants (female $n=11$, male $n=10$, mean age $46.7 \pm$ 10.6y) were recruited from the same geographical area to act as internal controls (NT). Participants were excluded if they suffered from hypertension (BP $\geq 140 / 90$ ) or were being medicated for the condition, a pre-study blood pressure recording was undertaken to confirm this. All participants completed a 4-day food diary in week 1 including 3 mid-week days and 1 weekend day, this was repeated in week 4 . The food diary was 
adapted from The Medical Research Council Collaborative Centre for Human Nutrition Research, National Diet and Nutrition Survey [26]. Although classified as an estimated food diary, food could be weighed and food labels submitted, in addition to photos being provided to establish portion size. All food diaries were recorded between January and March. An external control of the general UK population (GP) aged 19y and over was established from data provided by the National Diet and Nutrition Survey (NDNS) [23]. The two control groups were measured against each other to ensure homogeneity between groups to validate the food diary analysis and no statistically significant difference was found between the external observed and internal control groups for both the males $(p=0.6)$ and females $(p=0.12)$, except for the fact that the internal control group's mean intake of Mg was more than the RNI (See Table 1).

All diaries were analysed using Dietplan 6 (Forestfield software Ltd. West Sussex UK). Data were analysed for skewness and kurtosis and one way ANOVA used for interpretation of differences using SPSS (Version 22, IBM New York, USA).

Average daily magnesium intake established from the 8 day food diaries were compared between the HT, NT and GP groups and these were compared to both the UK RNI and the USA RDA to establish differences amongst the hypertensives and the recommendations.

The magnitude of the differences between observed and control groups were expressed as the effect size using Cohen's $d$, calculated as the absolute difference between means divided by the pooled $S D$ [27]. Qualitative descriptors of the effect size were as follows: negligible $(\mathrm{d}<0.19)$, small $(\mathrm{d}=0.20-0.49)$, moderate $(\mathrm{d}=0.50-0.79)$, or large $(\mathrm{d}>0.8)$.

\section{Results}

Mean Mg intake of the hypertensive males (HT) was compared to that of the normotensive male controls (NT) and the general UK population (GP) ( $90 \%$ of the RNI) as well as the RNI (Figure 1). A significant difference in dietary magnesium intake was seen between the HT group and the GP group $(\mathrm{p}<0.0001)$ and the RNI $(\mathrm{p}<0.0001)$. The observed group did not show a significant difference in intake compared to the internal control group, although a trend could be seen $(p=0.14)$ with a moderate effect size of 0.6 shown between the groups.

Mean $\mathrm{Mg}$ intake of the hypertensive females was compared against that of normotensive females, the general population and the RNI (Figure 2). The HT group had a

Table 1. Mean Mg intakes (mean \pm SD) for all participants $(H T+N T)$ stratified by age group (n = number of participants).

\begin{tabular}{cl}
\hline Age range & All Mg $(\mathrm{mg} / \mathrm{d})$ \\
\hline $19-50 \mathrm{y}$ & $244 \pm 65(\mathrm{n}=17)$ \\
$51-64 \mathrm{y}$ & $259 \pm 79(\mathrm{n}=16)$ \\
$65+\mathrm{y}$ & $200 \pm 45(\mathrm{n}=14)$ \\
All & $236 \pm 69(\mathrm{n}=47)$ \\
\hline
\end{tabular}


significantly lower mean dietary Mg intake than the RNI $(\mathrm{p}=0.006)$ and the NT group $(p=0.006)$. However, there was no significant difference between the HT group and the GP $(p=0.264)$. An effect size of 0.4 was found between the HT group and the GP control corroborating with the lack of significance between these two groups.

When total data (hypertensive and normotensive together) for both males and females were analysed, a one way ANOVA showed that there was a significant difference between the age groups $(\mathrm{p}=0.042)$ (Table 1$)$.

In females there is a reduction of over $100 \mathrm{mg} /$ day between all participants (both HT and NT) aged 51 - $64 \mathrm{y}$ and those aged $65 \mathrm{y}$ and above giving a 37\% reduction in intake.

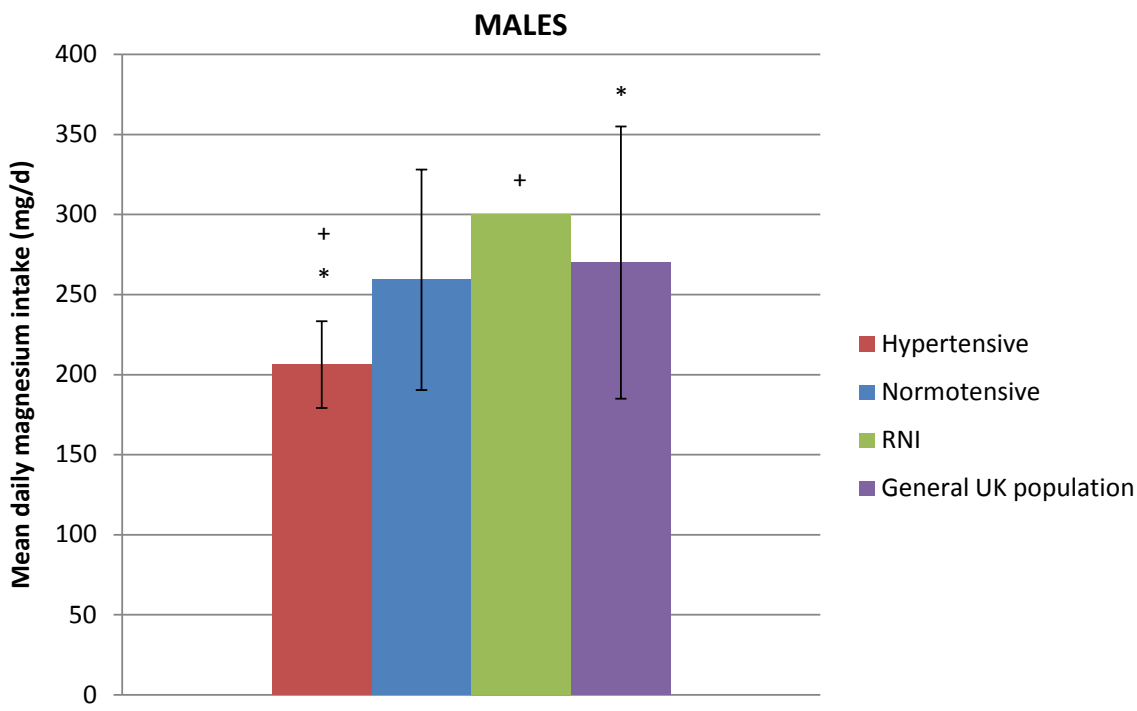

Figure 1. Mean + /- SD male daily magnesium intake comparing the hypertensive, normotensive, general UK population and the RNI (+denotes significant difference between observed and RNI, *denotes significant difference between HT and the GP with $\mathrm{p} \leq 0.05$ ).

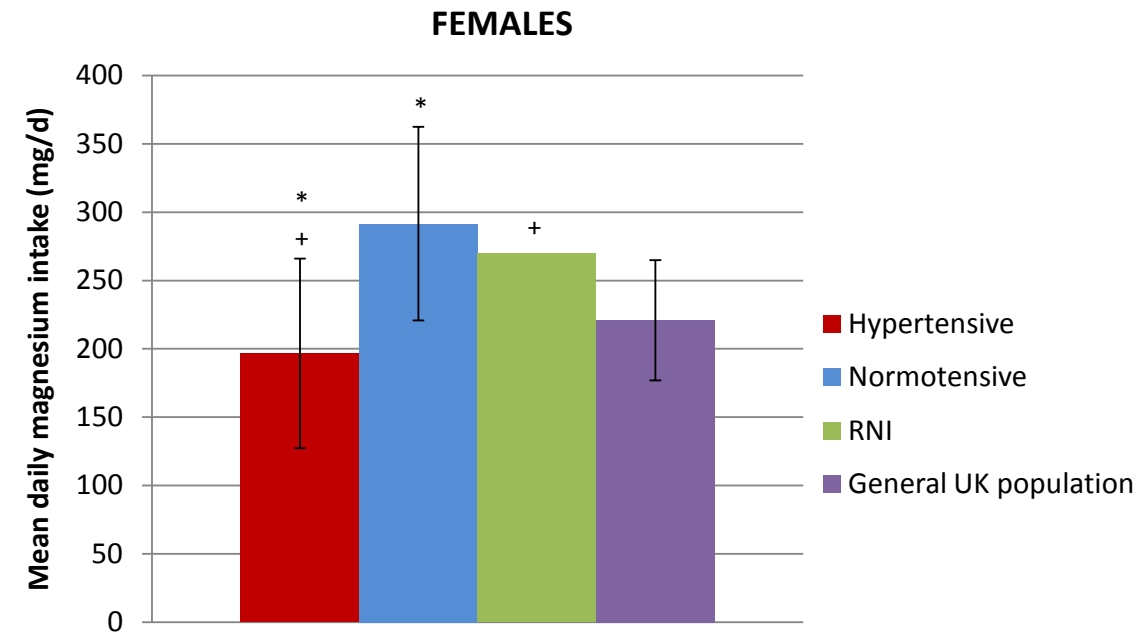

Figure 2. Mean $( \pm \mathrm{SD})$ female daily magnesium intake comparing the observed, internal control, external control groups with the RNI, (+denotes significant difference between observed and RNI, * denotes significant difference between HT and NT ( $\mathrm{p} \leq 0.05)$. 
For males the greatest difference was $31 \mathrm{mg}$ between the $18-50 \mathrm{yr}$ and $65+$ yrs population groups (Figure 3).

Male UK data for both the HT and NT was compared against the USA RDA (Figure 4). A significant difference in mean daily magnesium intake was shown when compared to the USA RDA $(420 \mathrm{mg})$ for both groups ( $\mathrm{p} \leq 0.001)$.

In females, there was a significant difference between the mean daily $\mathrm{Mg}$ intake of the hypertensive group and the USA RDA ( $\mathrm{p} \leq 0.001$ ); however, the normotensives did not show a significant difference to the USA recommendations $(p=0.238)$ (Figure 5).

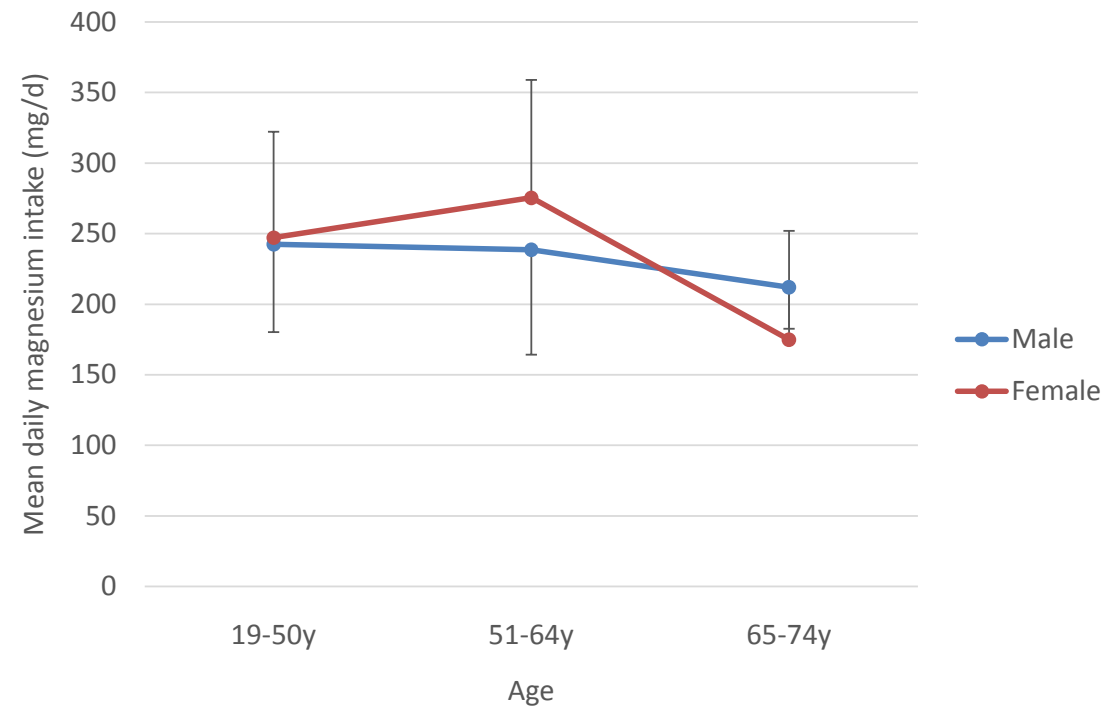

Figure 3. Mean daily dietary Mg intake for both males and females, $(\mathrm{HT}+\mathrm{NT})$ decreases with age. Error bars showing SEM.

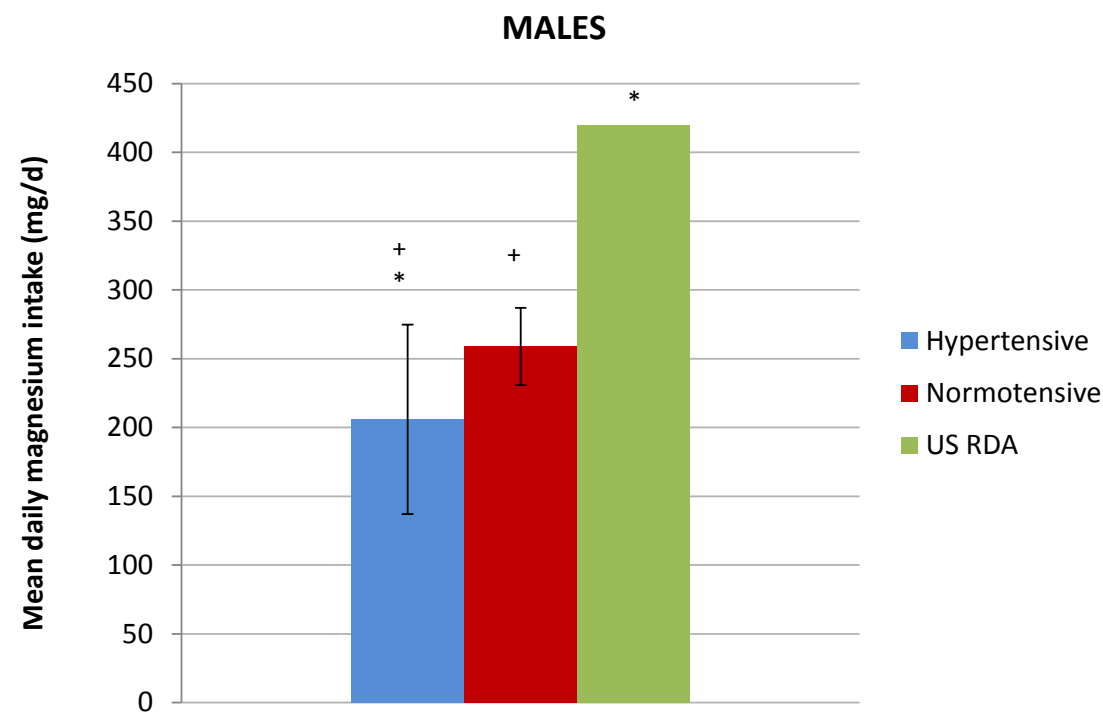

Figure 4. Male mean daily magnesium intakes (mean \pm SD) for HT and NT groups compared with the USA RDA (+denotes significant difference between HT and USA RDA, *denotes significant difference between HT and NT of $\mathrm{p} \leq 0.05$. 


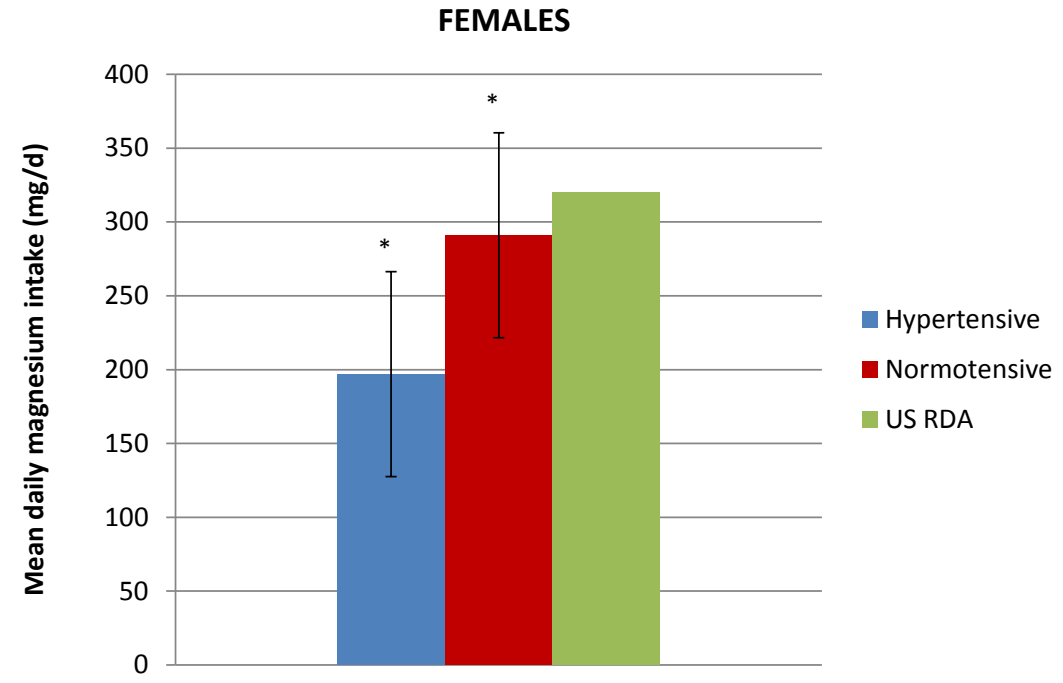

Figure 5. Female mean daily magnesium intake (mean $\pm \mathrm{SD}$ ) for HT and NT against USA RDA (*denotes significance of $\mathrm{p} \leq 0.05$. This can be seen between the HT and the USA RDA).

The UK general adult population's mean daily $\mathrm{Mg}$ intakes of $270 \mathrm{mg}$ and $221.4 \mathrm{mg}$ ( $90 \%$ and $82 \%$ of the RNI) for males and females respectively, represented $64 \%$ and $69 \%$ of the USA RDA.

\section{Discussion}

The primary finding from the current study is that hypertensive females appear to have a significantly lower intake of dietary magnesium than normotensive females. Both male and female hypertensives appear to have low dietary Mg intake when compared to both the RNI and RDA.

Analysis based on individual sexes elicited different results with observed males demonstrating a significantly lower daily dietary magnesium intake compared to both the GP and the RNI. The daily intake of the HT group was $94 \mathrm{mg} /$ day less than the RNI and $54 \mathrm{mg} /$ day less than the general population, considered to be a healthy population group. The normotensives demonstrated a trend (as evidenced by a moderate effect size of 0.8 ) towards a difference in daily intake although this was $11 \mathrm{mg} /$ day less than the general population and therefore significance was expected. The large standard deviation may have affected the statistical results but in real terms both the NT and GP had a similar intake in males.

Females showed a significant difference between both the NT and the RNI although the GP did not show a significant difference compared with the HT group. The GP was only $82 \%$ of the RNI, a low value which was also seen in the HT group's daily intake. However, female NT's mean daily intake of magnesium was above the RNI, the only group to have met this recommendation. As the NT groups are considered normotensive, it can be suggested that low magnesium intake ( $82 \%$ of the RNI), does not have a negative effect on blood pressure; however, the reduction of a further $24 \mathrm{mg} /$ day as 
found in the HT group may be important in explaining hypertension. It would be of interest to see if the GP group developed hypertension over time due to their low magnesium intake and thought must also be given to the previously mentioned fact that $25 \%$ of the general population in the UK is thought to be hypertensive [25].

In this study, the difference between Mg intakes of the HT and NT groups was small, however, the GP is already below the RNI suggesting that when this further decrease in $\mathrm{Mg}$ intake is seen the long term consequence on BP could be even greater.

When mean male intake of $206 \mathrm{mg}$ for the HT group across all ages is compared to the RNI the hypertensive patients have a significantly lower intake than recommended. Furthermore, the NDNS [26] states that generally males are at approximately $90 \%$ of the RNI, The NT group of healthy individuals were actually at $96 \%$, supporting these findings. Although both the HT and the NT group were below the RNI, NDNS [26] data used for the GP group showed that only $5 \%$ and $7 \%$ of the cohort (male and female respectively) had hypertension between the ages 19 - $64 \mathrm{y}$, increasing to $36 \%$ and $33 \%$ for the $65 \mathrm{y}+$ group. This results in mean hypertension of 21 and $20 \%$ for males and females in total, slightly below the established norm according to Public Health England [25]. This group (the GP) was at $90 \%$ of the RNI but below the expected norm for prevalence of hypertension. It can be suggested that $\mathrm{Mg}$ intake at $90 \%$ of the RNI has a small effect on blood pressure reinforcing previously published work showing a reduced magnesium intake is correlated with increased blood pressure [6] [28] However, the HT male group consumed just $69 \%$ of the RNI implying that a lower intake may have an effect on blood pressure.

For females the HT group's mean daily Mg intake was at $196.6 \mathrm{mg} /$ day equating to $72 \%$ of the RNI, while the NT group was above the RNI. Again this may suggest that a daily magnesium intake at $72 \%$ of the RNI may negatively affect blood pressure in adult women.

The NT group of healthy individuals for the North and East Herts NHS area were at $96 \%$ and $131 \%$ of the RNI compared to $90 \%$ and $82 \%$ for the general population showing that internal validity was higher in males than females. The high intake of dietary magnesium in the female NT group was unusual but the cohort was quite small and this over consumption only equated to $21 \mathrm{mg}$ /day above the RNI. The Mg intake figures are also means and therefore it is expected that about half of the subjects will fall above this mean value and half will fall below. However, the small female cohort and the high standard deviation could both be considered limitations of this work.

Previous research has shown that magnesium supplementation can have a positive effect on blood pressure [6] [28] [29], however the research in the UK is very limited. Bain et al. [29], using a UK population base found a greater association between males with low magnesium intake and risk of stroke, for which blood pressure is often a precursor, than in females. However, there is very little research which has examined $\mathrm{Mg}$ intake in the UK with consequences for blood pressure. Furthermore, until now, there have been no studies observing the habitual dietary intake of those with high blood pressure in the UK. Choi and Bae (2015) [15] found that there were a higher percentage 
of hypertensive males across all dietary magnesium intakes compared to females in the Korean population and Itoh (1997) [30] found a blood pressure decrease in 33 healthy Japanese who supplemented with magnesium. However Dickinson et al. (2009) [9] writing for the Cochrane Review used extensively for recommendations to the medical profession in the UK, did not find strong evidence for $\mathrm{Mg}$ reducing blood pressure. This study hopes to address this matter and move towards showing an association between those with hypertension and habitual dietary intake low in magnesium.

In the USA the RDA, set at 320 and $420 \mathrm{mg} / \mathrm{d}$ for females and males respectively, is higher than the RNI of the UK. As a comparison, the data from this UK study was also compared with the USA RDA and the results showed a significantly lower intake than recommended by the USA for all population groups. The UK general population's mean intake of $270 \mathrm{mg}$ and $221.4 \mathrm{mg}$ (90\% and $82 \%$ of the RNI) for males and females respectively, represented only $64 \%$ and $69 \%$ of the USA RDA.

Previous research has shown that $48 \%$ of the general population in the USA was below the RDA in 2005-2006, with rising calcium to magnesium ratios implicated in the rise of diabetes and stroke over the past 20 years [3]. Touyz [31], stated that magnesium may reduce arterial thickening and abnormal vascular tone as well as endothelial dysfunction linked with cardiovascular disease and high blood pressure. Therefore, a magnesium deficiency may play a role in the pathophysiological processes underlying blood pressure elevation.

With the exception of females in the $51-65$ y age group, all age groups consumed $\mathrm{Mg}$ below the RNI. The lowest intake could be seen in the $>65 \mathrm{y}$ age groups. In this age group, blood pressure increases become more prevalent due to decreases in arterial compliance and changes in the endothelial cells. Consideration should be given as to whether supplementation of this micronutrient might help reduce blood pressure increases normally seen in the older population group.

Although the USA has set the RDA at a higher level than the UK, the real issue lies with dietary intake and not with the recommendations. Initiatives to increase knowledge and awareness of this micronutrient may help to reduce blood pressure in the UK, subsequently saving the NHS money on costly drug intervention. Although no conclusions can be drawn on the mechanistic role of magnesium in prevention of hypertension, the current study does provide evidence that further investigation is warranted on the role of dietary magnesium intake in this area.

\section{Acknowledgements}

The authors are grateful to Professor Diana Gorog of the East and North Herts NHS Trust for her invaluable support with subject recruitment and to Bradley Fleming for his assistance with data collection.

\section{Ethical Approval}

It was granted through the NHS Research Authority, NRES Committee South CentralSouthampton A and the investigation was therefore performed in accordance with the 
ethical standards laid down in the 1964 Declaration of Helsinki and its later amendments.

\section{References}

[1] Zhang, X., Del Gobbo, L.C., Hruby, A., et al. (2016) The Circulating Concentration and 24-h Urine Excretion of Magnesium Dose- and Time-Dependently Respond to Oral Magnesium Supplementation in a Meta-Analysis of Randomized Controlled Trials. Journal of Nutrition, 146, 595-602. https://doi.org/10.3945/jn.115.223453

[2] Rylander, R. (2012) Magnesium Intervention and Blood Pressure-A Study on Risk Groups. Open Journal of Preventive Medicine, 2, 23-26. https://doi.org/10.4236/ojpm.2012.21004

[3] Rosanoff, A., Weaver, C.M. and Rude, R.K. (2012) Suboptimal Magnesium Status in the United States: Are the Health Consequences Underestimated? Nutrition Reviews, 70, 153164. https://doi.org/10.1111/j.1753-4887.2011.00465.x

[4] Ma, J., Folsom, A., Melnick, S., et al. (1995) Associations of Serum and Dietary Magnesium with Cardiovascular Arterial Thickness: The ARIC Study. Journal of Clinical Epidemiology, 48, 927-940.

[5] Volpe, S.L. (2013) Magnesium in Disease Prevention and Overall Health. Advances in $\mathrm{Nu}$ trition, 4, 378S-383S. https://doi.org/10.3945/an.112.003483

[6] Kass, L., Weekes, J. and Carpenter, L. (2012) Effect of Magnesium Supplementation on Blood Pressure: A Meta-Analysis. European Journal of Clinical Nutrition, 66, 411-418. https://doi.org/10.1038/ejcn.2012.4

[7] Jee, S.H., Miller, E.R., Guallar, E., et al. (2002) The Effect of Magnesium Supplementation on Blood Pressure: A Meta-Analysis of Randomized Clinical Trials. American Journal of Hypertension, 15, 691-696. https://doi.org/10.1016/S0895-7061(02)02964-3

[8] Burgess, E., Lewanczuk, R., Bolli, P., et al. (1999) Magnesium and Calcium. Canadian Medical Association Journal, 160, 35-45.

[9] Dickinson, H.O., Nicolson, D., Campbell, F., et al. (2009) Magnesium Supplementation for the Management of Primary Hypertension in Adults (Review).

[10] Rosanoff, A. and Plesset, M.R. (2013) Oral Magnesium Supplements Decrease High Blood Pressure (SBP > 155mmHg) in Hypertensive Subjects on Anti-Hypertensive Medications: A Targeted Meta-Analysis. Magnesium Research, 26, 93-99.

[11] Kass, L.S., Skinner, P. and Poeira, F. (2013) A Pilot Study on the Effects of Magnesium Supplementation with High and Low Habitual Dietary Magnesium Intake on Resting and Recovery from Aerobic and Resistance Exercise and Systolic Blood Pressure. Journal of Sports Science and Medicine, 144-150.

[12] Sanjuliani, A.F., de Abreu Fagundes, V.G. and Francischetti, E. (1996) Effects of Magnesium on Blood Pressure and Intracellular Ion Levels of Brazilian Hypertensive Patients. International Journal of Cardiology, 56, 177-183. https://doi.org/10.1016/0167-5273(96)02716-7

[13] Rosanoff, A. (2010) Magnesium Supplements May Enhance the Effect of Antihypertensive Medications in Stage 1 Hypertensive Subjects. Magnesium Research, 23, 27-40.

[14] Chidambaram, N., Sethupathy, S., Saravanan, N., et al. (2014) Relationship of Sodium and Magnesium Intakes to Hypertension Proven by 24-Hour Urianalysis in a South Indian Population. The Journal of Clinical Hypertension, 16, 581-586.

https://doi.org/10.1111/jch.12361 
[15] Choi, M.-K. and Bae, Y.J. (2015) Association of Magnesium Intake with High Blood Pressure in Korean Adults: Korea National Health and Nutrition Examination Survey 20072009. PLoS ONE, 10, e0130405. https://doi.org/10.1371/journal.pone.0130405

[16] Fox, C., Ramsoomair, D. and Carter, C. (2001) Magnesium: Its Proven and Potential Clinical Significance. Southern Medical Journal, 94, 1195-1201. https://doi.org/10.1097/00007611-200112000-00013

[17] Nakajima, T. (1997) Extracellular $\mathrm{Mg}^{2+}$ Inhibits Receptor-Mediated $\mathrm{Ca}^{2+}$-Permeable NonSelective Cation Currents in Aortic Smooth Muscle Cells. European Journal of Pharmacology, 320, 81-86. https://doi.org/10.1016/S0014-2999(96)00873-4

[18] Suzuki, M., Willcox, D.C., Rosenbaum, M.W. and Willcox, B.J. (2010) Oxidative Stress and Longevity in Okinawa: An Investigation of Blood Lipid Peroxidation and Tocopherol in Okinawan Centenarians. Current Gerontology and Geriatrics Research, 2010, Article ID: 380460. https://doi.org/10.1155/2010/380460

[19] Touyz, R.M., Laurant, P. and Schiffrin, E.L. (1998) Effect of Magnesium on Calcium Responses to Vasopressin in Vascular Smooth Muscle Cells to Spontaneously Hypertensive Rats. Journal of Pharmacology and Experimental Therapeutics, 284, 998-1005.

[20] Ohira, T., Peacock, J.M., Iso, H., et al. (2009) Serum and Dietary Magnesium and Risk of Ischemic Stroke: The Atherosclerosis Risk in Communities Study. American Journal of Epidemiology, 169, 1437-1444. https://doi.org/10.1093/aje/kwp071

[21] Joffres, R. (1987) Relationship of Magnesium Factors to Blood Pressure : Intake and Other Dietary the Honolulu.

[22] Townsend, M.S., Fulgoni, V.L., Stern, J.S., Adu-Afarwuah, S. and McCarron, D.A. (2005) Low Mineral Intake Is Associated with High Systolic Blood Pressure in the Third and Fourth National Health and Nutrition Examination Surveys: Could We All Be Right? American Journal of Hypertension, 18, 261-269. https://doi.org/10.1016/j.amjhyper.2004.09.017

[23] Public Health England (2014) National Diet and Nutrition Survey Results from Years 1, 2, 3 and 4 (Combined) of the Rolling Programme (2008/2009-2011/2012) Executive Summary.

[24] Institute of Medicine and Food and Nutrition Board (1997) Dietary Reference Intakes: Calcium, Phosphorus, Magnesium, Vitamin D and Fluoride.

[25] Public Health England (2014) Long Term Health Conditions.

[26] Medical Research Council. National Diet and Nutrition Survey. http://www.dapa-toolkit.mrc.ac.uk/documents/en/Foo/Food_diary_Adults_A5_Instruction s_v2_Q4.pdf

[27] Cumming, G. (2012) Understanding the New Statistics. Routledge, New York.

[28] Zhang, X., Li, Y., Del Gobb, L., et al. (2016) Effects of Magnesium Supplementation on Blood Pressure A Meta-Analysis of Randomized Double-Blind Placebo-Controlled Trials. Hypertension, 68, 324-333. https://doi.org/10.1161/HYPERTENSIONAHA.116.07664

[29] Bain, L., Myint, P., Jenning, A. and Lentjes, M. (2013) Dietary Magnesium Intake and Blood Pressure in an Adult British Population. Proceedings of the Nutrition Society, 72, E237. https://doi.org/10.1017/s0029665113002620

[30] Itoh, K., Kawasaka, T. and Nakamura, M. (1997) The Effects of High Oral Magnesium Supplementation on Blood Pressure, Serum Lipids and Related Variables in Apparently Healthy Japanese Subjects. British Journal of Nutrition, 78, 737-750. https://doi.org/10.1079/BJN19970191

[31] Touyz, R.M. (2003) Role of Magnesium in the Pathogenesis of Hypertension. Molecular Aspects of Medicine, 24, 107-136. https://doi.org/10.1016/S0098-2997(02)00094-8 
Submit or recommend next manuscript to SCIRP and we will provide best service for you:

Accepting pre-submission inquiries through Email, Facebook, LinkedIn, Twitter, etc. A wide selection of journals (inclusive of 9 subjects, more than 200 journals)

Providing 24-hour high-quality service

User-friendly online submission system

Fair and swift peer-review system

Efficient typesetting and proofreading procedure

Display of the result of downloads and visits, as well as the number of cited articles

Maximum dissemination of your research work

Submit your manuscript at: http://papersubmission.scirp.org/

Or contactwjcd@scirp.org 